\title{
On Carrier Diffusion in Nanostructures for Nanomedicine
}

\author{
Paolo Di Sia ${ }^{1-3}$ \\ ${ }^{1}$ University of Padova, School of Medicine, Department of Neuroscience, \\ Via Giustiniani 2, 35128 Padova, Italy \\ ${ }^{2}$ E-mail address: paolo.disia@gmail.com \\ ${ }^{3}$ Personal Web-page: www.paolodisia.com
}

\begin{abstract}
Nanometer-sized particles have optical, magnetic, chemical and structural properties setting them apart from bulk solids, with fruitful potential applications in nanomedicine. Carrier diffusion at the nanoscale plays a very important role in the dynamics of the processes taking place at this scale. Advances in analytical modelling allow a detailed study of carrier diffusion in the classical, quantum and relativistic case. Important results and previsions come by a promising analytical "timedomain" model for classical and quantum-relativistic transport in nano-systems, called DS model; it is demonstrating high generality and very good fitting with available experimental data of literature.
\end{abstract}

Keywords: Nanomedicine; Nanostructures; Nanophysics; Diffusion; Analytical Modelling; Quantum-Relativistic Effects; DS Model.

\section{REFERENCES}

[1] P. Di Sia, Quantum-Relativistic Nanodiffusion, Preprint DOI: 10.31219/osf.io/c3f8e (Dec 2019).

[2] P. Di Sia, Quantum-Relativistic Effects at the Nanolevel: Analysis of the Mean Square Deviation of Position with a new Analytical Model, Preprint DOI: 10.31219/osf.io/6r2bg (Dec 2019).

[3] P. Di Sia, Present and Future of Nanotechnologies: Peculiarities, Phenomenology, Theoretical Modelling, Perspectives, Reviews in Theoretical Science, 2(2), 146-180 (2014), DOI: https://doi.org/10.1166/rits.2014.1019.

[4] P. Di Sia, An analytical transport model for nanomaterials, Journal of Computational and Theoretical Nanoscience, 8, 84-89 (2011).

[5] P. Di Sia, An analytical transport model for nanomaterials: the quantum version, Journal of Computational and Theoretical Nanoscience, 9, 31-34 (2012).

[6] P. Di Sia, Nanotechnology between Classical and Quantum Scale: Applications of a new interesting analytical Model, Advanced Science Letters, 5, 1-5 (2012).

[7] C. A. Schmuttenmaer, Using Terahertz Spectroscopy to Study Nanomaterials, THZ Science and Technology, 1(1), 1-8 (2008).

[8] F. Borondics, K. Kamarás, M. Nikolou, D. B. Tanner, Z. H. Chen, A. G. Rinzler, Charge dynamics in transparent single-walled carbon nanotube films from optical transmission measurements, Physical Review B, 74, 045431-045436 (2006). 
[9] P. Di Sia, About the Influence of Temperature in Single-Walled Carbon Nanotubes: Details from a new Drude-Lorentz-like Model, Applied Surface Science, 275, 384-388 (2013).

[10] P. Di Sia, Relativistic nano-transport and artificial neural networks: details by a new analytical model, International Journal of Artificial Intelligence and Mechatronics (IJAIM), 3(3), 96-100 (2014).

[11] P. Di Sia, Quantum-Relativistic Velocities in Nano-Transport, Applied Surface Science, 446, 187-190 (2018), DOI: https://doi.org/10.1016/j.apsusc.2018.01.273.

[12] P. Di Sia, On surface electron mobility of nanomaterials for cancer nanotechnology, International Journal of Applied and Advanced Scientific Research (IJAASR), 3(2), 46-50 (2018); DOI: http://doi.org/10.5281/zenodo.2521284.

[13] P. Di Sia, Analytical Nano-Modelling for Neuroscience and Cognitive Science, Journal of Bioinformatics and Intelligent Control, 3(4), 268-272 (2014), DOI:10.1166/jbic.2014.1097.

[14] P. Di Sia, Mathematics and Physics for Nanotechnology - Technical Tools and Modelling, CRC Press \& Francis Group LLC (2019), https://www.crcpress.com/Mathematics-andPhysics-for-Nanotechnology-Technical-Tools-and-Modelling/Sia/p/book/9789814800020.

[15] S. T. Park, A. H. Zewail, Relativistic effects in photon-induced near field electron microscopy, Journal of Physical Chemistry A, 116(46), 11128-11133 (2012), DOI: https://doi.org/10.1021/jp304534n.

[16] J. B. Baxter and C. A. Schmuttenmaer, Conductivity of ZnO Nanowires, Nanoparticles, and Thin Films Using Time-Resolved Terahertz Spectroscopy, Journal of Physical Chemistry B, 110, 25229-25239 (2006).

[17] J. B. Baxter, and C. A. Schmuttenmaer, Carrier Dynamics in Bulk ZnO. I. Intrinsic Conductivity Measured by Terahertz Time Domain Spectroscopy, Physical Review B, 80, 235206-1 (2009).

[18] S. L. Dexheimer (Ed.), Terahertz Spectroscopy: Principles and Applications, CRC Press Taylor \& Francis Group LLC (2008).

[19] M. Th Hassan, Attomicroscopy: from femtosecond to attosecond electron microscopy, Journal of Physics B: Atomic, Molecular and Optical Physics, 51, 032005 (2018).

[20] N. Jain, R. Jain, N. Thakur, B. P. Gupta, D. K. Jain, J. Banveer, S. Jain, Nanotechnology: a Safe and effective Drug Delivery System, Asian Journal of Pharmaceutical and Clinical Research, 3(3), 159-65 (2010).

[21] N. Gharbi, M. Pressac, M. Hadchouel, H. Szwarc, S. R. Wilson, and F. Moussa, [60]Fullerene is a Powerful Antioxidant in Vivo with No Acute or Subacute Toxicity, Nano Letters, 5(12), 2578-85 (2005).

[22] A. S. Basso, D. Frenkel, F. J. Quintana, F. A. Costa-Pinto, S. Petrovic-Stojkovic, L. Puckett, A. Monsonego, A. Bar-Shir,Y. Engel, M. Gozin, H. L. Weiner, Reversal of axonal loss and disability in a mouse model of progressive multiple sclerosis, Journal of Clinical Investigation, 118(4), 1532-43 (2008). 